\title{
Fondements théoriques du développement local : quels apports du capital social et de l'économie de proximité?
}

Valérie Angeon et Jean-Marc Callois

\section{(2) OpenEdition \\ Journals \\ Édition électronique \\ URL : http://journals.openedition.org/ei/890 \\ DOI : $10.4000 /$ ei.890 \\ ISSN : 2553-1891 \\ Éditeur \\ Association Économie et Institutions}

Édition imprimée

Date de publication : 1 décembre 2005

Pagination : 19-50

ISSN : 1775-2329

\section{Référence électronique}

Valérie Angeon et Jean-Marc Callois, «Fondements théoriques du développement local : quels apports du capital social et de l'économie de proximité ? ", Économie et institutions [En ligne], 6-7 | 2005, mis en ligne le 31 janvier 2013, consulté le 23 avril 2019. URL : http://

journals.openedition.org/ei/890 ; DOI : 10.4000/ei.890 


\title{
Fondements théoriques du développement local : quels apports du capital social et de l'économie de proximité ?
}

\author{
Valérie ANGEON ${ }^{1}$ et Jean-Marc CALLOIS ${ }^{2}$
}

\section{Introduction}

Depuis plus de deux décennies, la référence au développement local tend à s'imposer dans les discours de politique économique. Présenté comme un mode de développement alternatif, il traduit la volonté d'augmenter l'efficacité des politiques publiques en les rapprochant des agents concernés, principalement les acteurs locaux. Cette pratique a trouvé un écho favorable dans les territoires, axant leurs stratégies de développement sur la mise en valeur de ressources locales et s'appuyant sur des démarches volontaristes et endogènes.

$\mathrm{Si}$ les principes du développement local se sont continuellement ancrés dans les pratiques et progressivement traduits par des préconisations concrètes en matière de développement, le concept demeure encore peu stabilisé dans la littérature. Du point de vue théorique, on admet qu'un territoire peut produire du développement selon la manière dont il fonctionne et s'organise.

Les travaux sur le développement local identifient les dynamiques sociales comme vecteurs d'évolution des territoires. Le constat établi est que la qualité des partenariats locaux conditionne la capacité des agents à s'entendre et à s'organiser - bref, à se coordonner - pour atteindre des objectifs de long terme. On souligne ainsi le poids des expériences d'apprentissage collectif et de coopération dans le développement des territoires (Greffe, 2002).

L'appréhension des propriétés du lien social (nature, qualité et densité des relations) se rattache aux questions de coordination. L'examen de ces propriétés amène, en effet, à considérer l'existence de relations de solidarité, de confiance, de proximité entre les agents. Cette manière d'aborder la coordination locale trouve ses fondements théoriques, à la fois, dans les approches du capital social (Coleman, 1988 ; Putnam, 1993) et de l'économie de proximités (Bellet et al. 1993 ; Bellet et al. 1998 ; Gilly et Torre, 2000, Pecqueur et Zimmerman, 2004).

1 UMR METAFORT - POP'TER ENGREF, 24, Avenue des Landais BP 90054 63171 AUBIERE Cedex 9, Angeon@engref.fr,

2 UMR METAFORT - CEMAGREF, 24, Avenue des Landais BP 50085 - 63172 AUBIERE Cedex, Jean-Marc.Callois@cemagref.fr 
L'objectif de cet article est de montrer que la théorie du capital social et l'économie de proximités constituent deux programmes de recherche qui permettent de consolider de manière convergente et complémentaire la théorisation du développement local. L'approche du capital social appréhende, à travers l'analyse des liens sociaux (nature, densité), les différentes ressources mobilisables pour le développement local. L'économie de proximités, quant à elle, fournit un cadre d'analyse des différents modes d'interaction entre acteurs situés sur un territoire. Ces interactions peuvent être basées sur des relations de proximité physique (proximité géographique) mais aussi sur l'appartenance à des organisations identiques ou analogues (proximité organisationnelle) ou sur la similitude cognitive des individus (proximité institutionnelle).

Cet article se structure en deux temps. Après avoir présenté les principes du développement local (I), nous nous attacherons à montrer de quelle manière les théories du capital social et l'économie de proximité en éclairent la compréhension (II). L'approche développée se propose ainsi d'étoffer les bases théoriques du développement local. Elle montre que ce n'est qu'à travers une approche mettant l'accent sur l'analyse des formes sociales organisées que ces principes peuvent être explicités.

\section{LES PRINCIPES DU DEVELOPPEMENT LOCAL}

Depuis la décennie 1960, le développement local apparaît comme une démarche novatrice de développement. Sa transcription dans les pratiques concrètes de développement passe par son inscription territoriale. Est associée à cette idée la conviction que le développement peut s'inscrire à des échelles infranationales. Cela suppose, d'une part, une certaine reconnaissance de l'existence de différenciations et de disparités spatiales (en termes de richesse par exemple) et, d'autre part, que les leviers d'action peuvent être entrepris à l'échelle territoriale ou relever d'initiatives locales.

Cette conception du développement va à l'encontre des théorisations qui ne conçoivent les déséquilibres spatiaux que comme des phénomènes transitoires, du reste, spontanément corrigés. Par ailleurs, cette vision du développement centrée sur des mécanismes endogènes rompt avec les premières recommandations en matière d'aménagement du territoire en France prônant le rôle centralisateur de l'Etat auquel il revenait d'organiser le territoire et d'en régenter les modalités de développement.

En pratique, les réflexions sur le développement local sont nées dans les territoires ruraux, marginalisés, enclavés, oubliés par les mesures nationales de soutien à la croissance et d'aménagement. Elles se sont soldées par des efforts en termes d'initiatives et 
d'innovations visant à préserver ces territoires d'une déstructuration éventuelle. Ces démarches de développement local ont suscité un certain enthousiasme à la généralisation de ses principes. Sont mises en avant les prédispositions de la population locale à se mobiliser, à s'accorder sur des objectifs déterminés comme centraux pour le territoire et à s'organiser pour les atteindre. En bref, est soulignée l'importance des dynamiques sociales dans la mise en œuvre des projets de territoire.

Cet intérêt pour le développement local se traduit également dans la littérature. Toutefois, si les écrits sur la thématique se multiplient, ils ne débouchent pas pour autant sur un cadre théorique de référence. L'essentiel des contributions se limite à un éventail d'exemples empiriques rendant compte des principes du développement local mais restent peu étoffés du point de vue théorique (Pecqueur 1989 ; Teisserenc 2002; Greffe 2002). Aussi, dans la première partie de cette communication, nous rappellerons l'essence du développement local. Nous en relèverons les principaux éléments d'analyse (1) qui permettent d'en esquisser une théorisation. Ces principes mettent en évidence l'importance de mécanismes particuliers de coordination (2).

\subsection{Le développement local : logiques, outils, cadres d'action}

Le développement est couramment analysé comme un processus de transformation et d'évolution de long terme. Le terme local renvoie à la notion de territoire. S'interroger sur le développement local revient à appréhender la question de l'échelle pertinente à partir de laquelle s'opèrent ces transformations. S'interpeller de cette manière invite à considérer le territoire dans ses multiples dimensions : politique, administrative, identitaire, culturelle, de conduite d'actions (champ d'intervention des acteurs), etc.

Le développement local exprime depuis trois décennies un mouvement de prise en charge du territoire par les acteurs locaux. D'abord apparu comme une nécessité contrainte par la crise, il s'est peu à peu présenté comme un choix. Le développement local se caractérise ainsi par le passage d'une approche essentiellement thérapeutique - répondre aux crises - à une approche préventive visant à faciliter l'adaptation du tissu local (économique et humain) aux enjeux auxquels il se confronte (internes, externes). Il repose sur la mobilisation de nombreux acteurs, des dispositifs institutionnels particuliers, qui facilitant et renforçant les dynamiques établies, contribuent à augmenter la cohérence du territoire. Dans l'exercice de synthèse sur le développement local auquel nous nous livrons ici, nous nous attacherons à rendre compte de ces caractéristiques. 
Nous viserons dès lors à énoncer les logiques du développement local, à en décliner les outils et à définir les cadres de l'action locale.

\subsubsection{Les logiques du développement local}

Le paradigme du développement local repose sur la capacité d'acteurs locaux à s'organiser autour d'un projet c'est-à-dire se fédérer autour d'un objectif de développement commun en mobilisant les potentialités et les ressources existant sur un territoire. Cette définition revêt trois dimensions. (i) Elle souligne le caractère localisé, territorialisé des activités et des actions. (ii) Elle invite à considérer la temporalité de ces actions. Les actes finalisés, en effet, rendent compte de la faculté des agents à concevoir un avenir commun. Dans le cadre du développement territorial, cet objectif commun repose sur la valorisation de ressources. (iii) Enfin, elle exprime que le territoire résulte des interactions entre acteurs impliqués dans une démarche collective. Ces trois points seront successivement évoqués pour la compréhension des logiques du développement local.

- Des activités et des actions localisées

On s'accorde à l'idée que les principes d'action locale se sont historiquement imposés en réaction aux évolutions économiques. Le mouvement de globalisation, en bouleversant les modes de produire, a induit des transformations des activités productives sans pour autant nier la pertinence des déterminants locaux. Ainsi, par exemple, parallèlement aux pratiques de délocalisation et de production standardisée, s'est accentuée l'affirmation du local avec la mise en valeur de produits spécifiques et de processus de production territorialement ancrés.

Ces mutations font apparaitre un spectre de situations complexes où le territoire demeure un lieu important de recomposition des tissus industriels et économiques. C'est alors une représentation nouvelle du territoire - englobant tout un ensemble de questions jusqu'alors essentiellement abordées dans le seul cadre des réflexions industrielles (dimension des unités productives, flexibilité, coopération inter-firmes etc.) - qui est proposée. Elle souligne le déplacement des lieux et des unités d'analyse de la croissance, appréhendant le territoire comme forme d'organisation économique efficace. L'exemple des districts industriels et des systèmes productifs locaux (SPL) peut être cité à ce titre. Le succès de ces formes productives localisées réside dans leurs modalités d'organisation marquées par des traditions culturelles (i.e. savoirfaire) véhiculant des valeurs communes, favorisant l'initiative locale et reposant sur des normes de coopération entre agents. 
Des conclusions précédentes, il ressort que les logiques de développement local reposent sur l'adoption, par les acteurs, de stratégies de mise en valeur de ressources territoriales. Dans les développements qui suivent, nous tenterons de préciser quels mécanismes président à ce processus.

Le territoire est lieu de concentration de ressources. Ces ressources, utilisées dans le processus de production, définissent le potentiel d'offre territoriale. Les valoriser constitue un enjeu de taille pour le territoire. Par ce biais, en effet, le territoire parvient à différencier ou spécifier son offre, ce qui dans une dynamique de développement est gage d'avantages concurrentiels.

Ces ressources sont plurielles et de nature diverse (ColletisWahl et Pecqueur 2001, Peyrache-Gadeau et Pecqueur, 2002 ; Angeon et Caron 2004). Elles peuvent être composites (c'est-à-dire constituées par une variété d'éléments combinés de multiples façons), spécifiques (intrinsèques au territoire, non reproductibles et non cessibles), complexes (recouvrant plusieurs propriétés à la fois) et latentes. Par ailleurs, ces ressources peuvent être intentionnellement construites.

Le processus de construction sociale de ressources repose sur des dynamiques d'acteurs. C'est bien en effet de la capacité des acteurs à révéler, activer, qualifier ou re-qualifier les ressources dont il est question. Cela suppose que les acteurs s'engagent dans des démarches de coopération. La stratégie de valorisation de ressources peut alors être conçue comme le fruit de la coordination d'acteurs impliqués dans des démarches d'action collective.

- Action collective

Le développement local désigne une dynamique d'initiatives locales (privées ou publiques) qui met en mouvement des acteurs. Ces derniers qui se réunissent autour d'un projet - dont le principe de valorisation de ressources est l'essence - font collectivement par ce biais exister le territoire. L'élaboration de projets se concrétise à travers la mobilisation des acteurs autour d'une stratégie commune. Elle trouve sa traduction opérationnelle dans une programmation cohérente d'actions. Le développement local peut être ainsi assimilé à un cadre favorable à l'action collective au sein duquel le territoire se construit.

L'aboutissement de l'action collective suppose que les acteurs parviennent à s'entendre sur les objectifs visés et les moyens de les atteindre. La mise en cohérence des diverses représentations du territoire que portent les acteurs est, en effet, le garant d'une dynamique effective de coopération. Au sein d'un territoire, la 
coopération entre acteurs se matérialise à travers l'établissement de partenariats locaux. Ces réseaux d'acteurs locaux renforcent la cohésion sociale et favorisent la cohérence territoriale. Cette cohérence peut elle-même être renforcée et rendue plus efficace par un cadre institutionnel adapté.

\section{Quel cadre institutionnel pour les actions de développement local?}

L'intérêt reconnu aux démarches d'action collective locale s'est transcrit, en France, dans des cadres institutionnels particuliers. Des structures nouvelles ont, en effet, été imaginées (dont les plus connues sont les Etablissements Publics de Coopération Intercommunale et les Pays) qui constituent les nouveaux référentiels de l'action locale. Ces structures redéfinissent l'architecture institutionnelle en adoptant des procédures de mise en accord des acteurs.

Pour ce qui concerne l'exemple des pays, concrètement, les centres d'intérêts collectivement exprimés par les acteurs sont inscrits dans des chartes. Ce qui importe ici, c'est le caractère conventionnel de ces chartes. Il implique que les acteurs s'engagent dans une procédure de concertation. Il souligne l'existence d'un accord local (sur les principes et les finalités de l'action) indépendamment de toute référence au document formel matérialisant les engagements des partenaires.

Les chartes sont énoncées sous forme de contrats qui instituent les relations de partenariat entre acteurs. On s'arrête ici sur le caractère formel de la charte. On notera que l'engagement des agents dans un contrat nécessite qu'ils s'inscrivent dans des rapports de confiance mutuelle. Ces engagements sont volontaires. Il importe donc que chacun des protagonistes adhère à ces principes et partage une même vision du territoire, une conception commune de ses modalités ou potentialités de développement.

Ainsi, ces cadres institutionnels - matérialisés par la constitution d'une charte - visent à proposer des repères pour l'action. Ce sont, en ce sens, de véritables projets de territoire, c'està-dire qu'ils sont le support de démarches, stratégies ou initiatives pour le développement du territoire. A ce titre, ils définissent des objectifs à atteindre sous certaines conditions ou contraintes. Ces dernières renvoient au fait qu'existent - ou le cas échéant que soient stimulées - les ressources ${ }^{3}$ nécessaires à la formulation du projet. Un projet caractérise donc "la conjonction d'analyses, de désirs et de savoir-faire collectif qui permet de polariser l'action de chacun autour d'une ambition commune, de résister aux forces centrifuges,

\footnotetext{
3 Le terme ressource est entendu ici au sens large, c'est-à-dire au sens de "moyens" ou "potentialités".
}

24 Economie et Institutions $-n^{\circ} 6$ et $7-1^{\text {er }}$ et $2^{\text {e }}$ semestres 2005 
de surmonter les contradictions internes d'intérêts, de saisir les opportunités qui se présentent d'exploiter les marges de manœuvre, de replacer l'action de chacun improvisée en fonction d'événements aléatoires dans une perspectives à long terme" (Calame, 1991, p. 35).

Ces structures tendent à présenter le développement local comme une pratique institutionnalisée sous de multiples facettes. Fondée sur les logiques du développement local et expression d'un mode de gouvernance territoriale, elles sont lieux d'organisation de l'action collective. Loin de se limiter à n'être qu'un simple programme d'actions, le développement local repose sur la conviction que les acteurs du territoire sont capables de mettre en adéquation leurs initiatives, d'avoir une représentation commune de ses principaux enjeux, de ses atouts ou de ses fragilités. Une telle considération fait ressortir que les spécificités territoriales importent dans le développement.

L'approche du développement local s'affranchit ainsi des visions réductrices des théories économiques du développement qui considèrent l'espace local comme une entité neutre (ou réduite à un point). Elle fait apparaitre que les ressorts territoriaux facilitent la coordination, ce qui dans l'analyse des conditions du développement, revêt un caractère central.

\subsection{De l'importance des coordinations locales dans le développement 4}

Les principales conclusions de la section précédente nous amènent à admettre l'idée d'un développement situé dans l'espace. L'hypothèse avancée est que les dynamiques d'évolution différenciées qu'affichent les territoires sont expliquées par le comportement des individus-acteurs. Le faisceau de relations qu'entretiennent ces acteurs, les réseaux qu'ils mobilisent, le poids du temps long avec ses implications en termes d'apprentissage collectif, importent dans l'analyse des trajectoires des territoires. En conséquence, le territoire ne peut être postulé : il est le résultat de jeux d'acteurs en relation les uns avec les autres.

Dans cette section, nous nous livrons à une réflexion théorique sur le développement local. Cette réflexion nous invite à mettre l'accent sur les coordinations locales, facteurs de développement. On s'intéressera dès lors (i) à la nature de ces coordinations et (ii) aux effets qu'elles génèrent.

4 Cette section s'inspire d'une réflexion plus large menée dans le cadre d'un programme de recherche co-financé par le Ministère de l'Ecologie et du développement durable et le Ministère de l'équipement (PUCA). 


\subsubsection{La nature des coordinations dans le développement local}

Processus collectif de création ou d'innovation territoriale, le développement local tend à fédérer des acteurs autour d'un projet commun. Il met en évidence l'efficacité des relations entre les agents pour valoriser les richesses dont ils disposent. Ces modalités de coordination entre acteurs ne s'inscrivent pas nécessairement dans un cadre marchand.

Comme souligné précédemment, les dynamiques de développement local impliquent une conception partagée par les acteurs des enjeux du territoire. L'idée d'accord entre acteurs, énoncée comme condition nécessaire à l'expression d'un projet, n'est pas sans poser de questions sur la manière dont les intérêts individuels sont rendus compatibles. La mise en cohérence des intérêts privés ne relève pas, en effet, d'une harmonie spontanée et l'expression des intérêts privés peut parfois contrecarrer les logiques collectives.

L'analyse montre que président aux relations entre acteurs des systèmes de valeurs. Ces règles (entendues ici au sens large) normalisent leurs comportements. Elles ne sont pas nécessairement formalisées ; elles peuvent être tacites. Elles visent à concilier les intérêts individuels en ce qu'elles font converger les anticipations et les représentations des agents. De la capacité d'adhésion des individus à ces systèmes de représentation dépendent, en effet, la limitation de démarches individualistes et le succès de l'action collective.

Ces règles partagées correspondent à un ensemble d'"institutions invisibles" (North, 1990 ; Dupuy et Torre, 1998, 2000), parmi lesquelles on peut mentionner par exemple la confiance. Les rapports de confiance s'appuient sur les engagements mutuels que prennent les agents les uns vis-à-vis des autres. Ils facilitent leurs capacités d'anticipation (i.e. ils éclairent les autres sur leurs intentions d'action) et régulent leur liberté de conduite et d'action. Favorisant une meilleure compréhension entre les agents, encourageant la transparence et la circulation d'informations, ces liens de confiance facilitent la coopération. Ils permettent, en outre, de déboucher sur des régularités de comportement et préviennent les défections ou les comportements opportunistes. La confiance permet aux agents de forger leur espace de rapports. Elle contribue à stabiliser ou à renforcer les liens entre les agents en favorisant le développement de signes leur permettant de limiter les problèmes d'asymétrie d'information et d'incertitude.

La confiance, présentée alors comme un "lubrifiant des relations sociales" (Arrow, 1974), rend possible la répétition des actes de coopération et devient, ce faisant, une modalité de coordination entre acteurs. La fréquence des interactions entretient les conditions 
de mise en accord des acteurs du territoire sur la nature et l'orientation $\mathrm{du}$ projet qu'ils entendent mettre en œuvre. Les habitudes de coopération favorisent l'adoption par les acteurs de positions consensuelles. Ils parviennent, par ce biais, à contrer plus efficacement la survenue des problèmes et à s'accorder sur les priorités. Elles rendent compte de la capacité des agents à reconnaître des enjeux communs et à se les approprier. Ainsi, les institutions invisibles favorisent l'établissement de liens entre acteurs, amplifient les dynamiques de coopération et limitent les dissensions.

Le constat que la qualité des partenariats locaux conditionne la capacité des agents à s'entendre et à s'organiser pour atteindre des objectifs de long terme, souligne l'importance des coordinations locales dans le développement. A l'issue de ce qui précède, nous concevons que ces dynamiques sociales contribuent à une meilleure circulation de l'information et renforcent l'action collective.

\subsubsection{Les mécanismes impliquant les coordinations locales}

Soutenir que les coordinations locales ont une incidence sur le développement implique d'identifier les mécanismes auxquels elles renvoient. L'approche économique propose une grille d'analyse de ces mécanismes appréhendés comme des externalités. On démontre alors que les coordinations locales sont productrices d'externalités positives. On en distingue deux types. (i) Le premier se rattache à des questions de collecte et de circulation de l'information. (ii) Le second se rapporte à l'action collective.

- Une meilleure collecte et circulation de l'information

Les exemples sont nombreux où les agents économiques sont conduits à prendre des décisions inefficaces par manque d'informations. Les cas des marchés du travail et du crédit peuvent être cités, à ce titre. Ils illustrent des situations d'incomplétude ou d'asymétrie d'information formalisées notamment par la théorie de l'agence. Comme nous l'avons exposé précédemment, les liens qu'entretiennent les agents reposent sur un ensemble de règles qui facilitent leurs modalités d'organisation. Inscrits dans des rapports de confiance, ils sont incités à rendre transparentes les informations les concernant (caractéristiques intrinsèques des individus). Ces modalités de coordination locale favorisent non seulement une meilleure connaissance des comportements individuels mais permettent également un accès plus facile (et moins coûteux) à des données concernant leur environnement immédiat.

A ce titre, l'exemple du progrès technique dans le processus de production des entreprises peut être donné. Il met en évidence la qualité des coordinations locales dans le partage et la diffusion de 
l'information. Ces informations, qu'elles concernent la transmission d'une technique de production ou d'un apprentissage (savoir-faire), sont sources de compétitivité pour les entreprises. L'accès de ces firmes à de telles informations se solde par une meilleure connaissance de leur environnement économique. Cette dernière permet, en outre, aux firmes de réaliser des économies d'échelle. Les districts industriels et les SPL fonctionnent sur ce mode. Ils décrivent des configurations où le degré d'interaction entre des agents appartenant au même milieu socioculturel et ratifiant les mêmes normes de comportement - est élevé.

Un comportement opportuniste de la part d'entrepreneurs locaux peut conduire à dissimuler des informations sur l'état des marchés ou la technologie, alors qu'il serait collectivement efficace de les partager. S'agissant de la coordination d'investissements, un raisonnement analogue peut être tenu. Il peut être tentant individuellement de profiter des investissements réalisés par d'autres, ce qui aboutit à un niveau d'investissement inefficace. Ces investissements peuvent concerner non seulement des infrastructures collectives, mais également des éléments intangibles comme la réputation d'un territoire. En somme, le partage de l'information améliore la qualité des décisions par rapports à des procédures individuelles de choix. La meilleure circulation de l'information que favorisent les liens locaux s'avère dès lors avantageuse dans la mesure où elle contribue à réduire le risque, l'incertitude et à économiser les coûts de transaction.

Dans les projets de territoire, le principe de gouvernance rend bien compte d'une certaine efficience collective. Les agents, associés à la prise de décision, s'organisent sur le mode de la coopération. Amenés à partager des informations de diverse nature, ils détiennent une meilleure connaissance des individus et de leurs intentions ainsi que de leur environnement socio-économique. Par ce biais, ils améliorent la qualité de leurs choix, ce qui leur permet de prendre véritablement part au dispositif d'action et de maitriser les enjeux de leur territoire, plutôt que de subir des contraintes de choix imposées par d'autres.

Les modalités de coordination locale, en facilitant la procédure de décision et en améliorant la qualité des choix, produisent un impact positif sur le bien-être collectif. Les agents agissent, dès lors, dans un cadre proche de la situation optimale d'information pure et parfaite, malgré le fait que ce sont des mécanismes hors marché qui régissent les modalités de leur mise en relation. Cette meilleure compréhension des comportements individuels et de l'environnement renforcent les stratégies d'action collective, au cœur des logiques de développement local.

28 Economie et Institutions $-n^{\circ} 6$ et $7-1^{\text {er }}$ et $2^{\text {e }}$ semestres 2005 
- Le renforcement des logiques d'action collective

Les coordinations locales renforcent l'action collective. Le principe de l'action collective repose sur l'habileté des agents à mettre en commun des ressources pour atteindre des objectifs qui n'auraient pas été atteints individuellement. On convient ainsi à l'idée que l'action collective résulte des dynamiques de coopération qui s'établissent entre les agents.

L'analyse théorique montre qu'en situation de jeu répété, les agents sont susceptibles de développer un comportement coopératif. Si l'on se fie à l'observation des comportements effectifs des individus, on constate que la coopération résulte d'une combinaison de règles (valeurs, normes conventions, routines etc.). De manière plus intuitive, on se range à l'idée que l'occurrence des interactions amène les individus à développer des routines. Ces routines stabilisent les dynamiques d'interaction parce qu'elles permettent aux agents d'acquérir des informations essentielles visant à mieux comprendre et à anticiper leurs intentions réciproques d'action. Elles s'accompagnent d'autres phénomènes (i.e. la réputation, confiance, réciprocité) qui confortent l'espace relationnel. Les relations que nous décrivons ne sont pas exclusivement bilatérales. Les agents entretiennent en effet une pluralité de liens. L'individu est ainsi décrit au travers des relations qui l'unissent à d'autres dans un contexte donné.

Comme nous venons de l'évoquer, les comportements coopératifs demeurent au fondement de l'action collective. Ils mettent en évidence l'ensemble des relations interpersonnelles dans lesquelles s'inscrivent les agents. Dans les pratiques d'action locale, ces relations s'établissent en dehors du marché. Dans cet espace de proximité que constitue le territoire, les institutions invisibles qui procèdent à la coordination des agents placent ces derniers en situation de développer des liens s'apparentant à ceux que l'on observe dans des structures de type communautaire. Ces réseaux communautaires qui reposent sur une nature de liens particuliers entre agents (de solidarité, de coopération, de proximité), imposent dès lors une représentation complexe du territoire et de ses modalités de développement.

Les logiques de développement local reposent sur le principe de cohérence d'une micro-société. Elles rendent compte de ce que chaque territoire possède son histoire, ses potentialités, ses ressources et ses contraintes lesquelles se transmettent à travers des formes sociales organisées. Il apparaît dès lors nécessaire de s'intéresser aux éléments de théorisation concernant ces dernières pour une meilleure compréhension des mécanismes auxquels renvoie le développement local. 


\section{REGARD THEORIQUE SUR LE ROLE DES FORMES SOCIALES ORGANISEES DANS LE DEVELOPPEMENT LOCAL}

Cette partie examine successivement les deux approches théoriques retenues pour une formalisation du développement local: le capital social et l'économie de proximités. Le capital social renvoie à l'ensemble des éléments intangibles structurant les relations entre individus (réseaux sociaux, normes, confiance ...) et leur permettant d'accéder à des ressources imbriquées dans la structure sociale (Lin, 2001). Le programme de recherche que constitue l'économie de proximités (Bellet et al. 1993 ; Bellet et al. 1998 ; Gilly et Torre 2000 ; Pecqueur et Zimmerman 2004) analyse les processus d'interaction entre les agents. Les deux approches ont en commun, de traiter de manière complémentaire, la question de la densité des liens sociaux des acteurs d'un territoire qu'elles présentent selon les cas soit comme un potentiel soit comme un frein de développement. Il apparaît dès lors que l'articulation de ces deux programmes de recherche permettrait de mieux cerner les mécanismes à l'œuvre dans les processus de développement local.

\subsection{Economie de proximité et théories du capital social : quels fondements?}

Les théories du capital social lient la performance économique à l'état des relations sociales et au cadre institutionnel (Putnam, 1993, 1995 ; Knack et Keefer, 1997 ; Krishna, 2001). Dans la même perspective, l'économie de proximité explique le développement territorial par les dynamiques de proximité. Il faut entendre par là les interactions entre agents et le rôle des institutions formelles et informelles (Zimmermann et al. 1998).

2.1.1. L'économie de proximité : une approche centrée sur les questions d'interaction et de coordination des agents

Le concept de proximité apparaît dans la littérature avec les travaux de Bellet et al. (1992) et de Bellet et al. (1993), du groupe français "Dynamiques de proximité". Ce programme de recherche est né au début de la décennie 1990.

- La genèse du concept

Les travaux en économie de proximité s'inscrivent originellement au croisement de l'économie industrielle et spatiale. Le rapprochement entre ces deux disciplines fait l'objet d'un regain

30 Economie et Institutions $-n^{\circ} 6$ et $7-1^{\text {er }}$ et $2^{\text {e }}$ semestres 2005 
d'intérêt (depuis le début des années 1990) appréciable sous deux angles. Le premier est lié au développement de travaux en économie industrielle prenant en compte l'enracinement spatial des activités et plus largement des phénomènes économiques. L'espace compte et devient un élément explicatif et constitutif des mécanismes économiques ${ }^{5}$. Le deuxième angle à partir duquel peut être cerné le croisement entre économie industrielle et économie spatiale est relatif au traitement de problématiques d'économie industrielle dans l'analyse des phénomènes spatiaux (Pecqueur et Zimmerman, 2004). La prise en compte de ce recouvrement disciplinaire a alimenté un champ de recherche fécond que se sont appropriés les économistes de la proximité.

Et même si Bellet et al. (1992) soulignent que la notion de proximité n'a pas toujours été exprimée de manière explicite dans les travaux du début de la décennie, ils défendent l'idée que les phénomènes de proximité ne sont pas à nier et méritent une attention particulière. A partir de ce concept, les économistes de la proximité analysent les phénomènes d'organisation et montrent que ceux-ci se rapportent à l'établissement de relations entre agents. Ces dernières ne s'effectuent pas nécessairement au sein du cadre marchand. Le terme de proximité traduit l'importance générale accordée aux interactions entre agents.

On notera toutefois que ce programme de recherche ne provient pas d'un strict cadre théorique (Gilly et Torre, 2000), et que la mise en valeur des phénomènes de proximité varie selon les travaux développés (Rallet, 1999). Les fondateurs du programme affichent en effet un certain éclectisme théorique, s'inscrivant dans des champs théoriques aussi divers que : l'économie des conventions, l'économie institutionnaliste, l'économie des contrats, l'économie des coûts de transaction.

Le concept de proximité renvoie à une dénomination plurielle (Bellet et al. 1998, Rallet 1999). Le terme est d'un usage courant et recouvre diverses notions. On parle en effet de : relations de proximité, emplois de proximité, services de proximité, justice de proximité, etc. Par ailleurs, la proximité se décline selon divers registres ou ordres. Les termes de proximité géographique, organisationnelle, relationnelle, matérielle, territoriale, institutionnelle, etc., se côtoient dans la littérature. Certains contributeurs (Grossetti, 1998 notamment, de tradition sociologique) retiennent trois ordres de proximité. Ils distinguent :

La proximité matérielle : elle est relative à l'espace physique (l'unité métrique étant un critère de mesure objectif), mais comprend également l'idée de l'espace socialement construit. Dans cette

5 Cf. Rallet et Torre (1995) pour une synthèse de l'ensemble de ces travaux. 
dernière optique, on se range à l'idée que l'espace est modelé, façonné par l'action humaine.

La proximité sociale : elle caractérise l'appartenance à des positions sociales. Elle fait référence à l'espace social des individus. "Cette proximité est une ressemblance, une similarité, elle n'implique nullement des échanges" (Grossetti, 1998, p. 89).

La proximité relationnelle : elle traite des échanges effectifs entre les agents. Elle associe les deux formes de proximité précédentes. Elle est à la fois sociale "puisqu'elle est fondée sur des critères proprement sociaux, et matérielle, puisqu'elle implique des échanges directs (au moins pour les éléments les plus proches) et donc un cadre physique d'interaction" (Grossetti, 1998, p. 90).

Dans la tradition économique, s'agissant de caractériser la structuration des relations individuelles ou sociales, les principaux contributeurs s'accordent à retenir deux déclinaisons analytiques de la proximité : la proximité géographique et la proximité organisée. Ces composantes de la proximité expriment respectivement la séparation géographique et économique des agents. On s'apercevra que d'une certaine manière ce découpage des types de proximité se recoupe avec le premier.

- La double dimension des interactions individuelles : proximité géographique et proximité organisée

(i) La proximité organisée traite de la séparation économique des agents. Elle identifie l'espace de rapports des individus. De ce point de vue, sont proches des acteurs qui appartiennent au même espace de rapports et entre lesquels s'établissent des interactions de nature différente (marchandes ou non marchandes, intentionnelles ou non intentionnelles, etc.). La proximité organisée renvoie également à l'espace de représentation des individus. Selon cette dernière acception, sont désignés proches des agents qui se ressemblent et qui partagent le même espace de valeurs, de règles, de modèles de pensées et d'actions.

En clair, la proximité organisée repose sur deux logiques ou recouvre deux dimensions : la logique d'appartenance et la logique de similitude. Dans la première dimension, c'est l'effectivité des relations qui est évoquée, alors que dans la seconde, c'est de l'adhésion à un même espace de référence dont il est question. Cette deuxième dimension de la proximité organisée (la logique de similitude) relève du domaine institutionnel. Elle est appelée par certains auteurs proximité institutionnelle et est considérée parfois comme un registre à part de la proximité.

(ii) La proximité géographique décrit la position relative des agents dans un espace plan déterminé. Elle renvoie à la localisation des interactions entre les agents. Elle rend compte de leur éloignement, et se mesure en termes de distance exprimée en fonction du temps et/ ou des coûts à supporter pour la franchir. 
Dans ces conditions, on conçoit que la proximité géographique n'est pas strictement naturelle ou physique, puisque l'espace physique subit des transformations humaines. Elle est à la fois support et construit d'interactions sociales. Cette forme de proximité fait référence à la notion d' "espace géonomique" de Perroux (1964), "renvoyant largement à la localisation des entreprises, elle intègre la dimension sociale des mécanismes économiques, ou ce que l'on appelle parfois la distance fonctionnelle" (Zimmermann, 1998, p. 9).

"La proximité géographique traduit la plus ou moins forte matérialité de l'espace: elle est traitée comme une contrainte pesant sur le développement des interactions entre agents. Dans l'analyse, elle joue le rôle d'une condition permissive : la proximité favorise a priori le développement des interactions entre agents. Elle facilite les échanges de produits mais aussi les rencontres, les échanges d'information, le partage des connaissances" (Rallet, 1999, p. 13). Selon cette assertion, la proximité géographique favorise l'établissement de liens entre les agents. Elle permettrait le développement de liens de type organisationnel. On peut aussi associer à la proximité géographique - lorsqu'elle se circonscrit à un espace précisément délimité et stable - à un lieu commun de représentations au sein duquel on identifie des aspects tels que la culture, les traditions, les valeurs, etc. Comprise en ce sens, la proximité géographique pourrait expliquer certaines ressemblances entre les agents en termes de représentation. Elle favoriserait ainsi la dimension institutionnelle de la proximité organisée.

A l'issue de ces propos, on convient du rôle de la proximité géographique dans la constitution des relations de face à face d'une part, et dans la diffusion de règles et de modèles d'actions communs d'autre part. En d'autre termes, la proximité géographique peut favoriser le renforcement des logiques d'appartenance et de similitude qui caractérisent la proximité organisée ${ }^{6}$. Comme en atteste la conclusion précédente, les deux types de proximité s'articulent.

2.1.2. L'approche théorique du capital social: une analyse des formes sociales organisées

Le concept de capital social n'est pas récent. S'il a été initialement proposé par les sociologues ${ }^{7}$ - dont les centres d'intérêt portent sur l'analyse des groupes sociaux et des institutions - les économistes se l'approprient depuis peu. Il faut attendre, en effet, en économie, le développement de travaux sur les coordinations non

6 La proximité géographique peut être parfois génératrice de conflits (Torre et Caron, 2002).

7 Les premiers travaux recensés dans la littérature remontent à Weber et Simmel. 
marchandes et de réflexions sur la prise en compte de l'environnement institutionnel et social dans les procédures de décision des agents, pour voir apparaître l'idée de capital social.

La plupart des travaux sur le capital social s'accordent sur ses effets positifs : il facilite la coordination des agents et semble être une condition de développement. Le lien entre capital social et développement a été approfondi dans la littérature ; le premier est présenté comme une variable explicative de la performance économique locale. Afin de caractériser le capital social, dans cette section, (i) nous aborderons quelques éléments de définition du concept et (ii) tenterons en second lieu d'en caractériser les formes.

- Le capital social, éléments de définition

Si la notion de capital social s'est largement diffusée en une vingtaine d'années, ce regain d'intérêt n'a toutefois guère contribué à stabiliser le concept. Ainsi, donner une définition du capital social reste un exercice difficile. Nombreuses sont les contributions qui définissent le terme. (i) Nous commencerons par proposer une définition qui recueille un certain consensus, (ii) avant de passer brièvement en revue les travaux de Coleman $(1988,1990)$ et (iii) de Lin (2001).

(i) Le capital social se définit comme l'ensemble des normes et des réseaux qui facilitent l'action collective (Woolcok et Narayan, 2000). Cette définition courante du capital social fait ressortir deux de ses dimensions essentielles. La première est liée aux normes et valeurs (ou ensemble de règles informelles) qui régissent les interactions entre agents. La seconde détermine le capital social par ses caractéristiques structurelles. Le capital social désigne alors, dans ce cas, le cadre formel au sein duquel s'établissent les relations entre les agents.

Ainsi défini, on conçoit le capital social comme l'ensemble des institutions (entendues ici au sens large c'est-à-dire règles ou cadres d'action des agents) formelles ou informelles qui facilitent la coopération antre acteurs en vue d'actions finalisées. Cette conception du capital social revient d'une certaine façon à intégrer les facteurs sociaux dans les principes d'action individuelle (Coleman, 1988).

(ii) Les travaux de Coleman sont parmi ceux qui constituent une référence incontournable sur le capital social. Pour l'essentiel, ses apports consistent à formuler une théorisation de l'action rationnelle centrée sur des déterminants sociaux. Coleman introduit le concept de rationalité en sociologie. Il présente le capital social comme une forme particulière de capital qui rend possible l'action sociale. Le capital social est donc à l'origine des relations développées entre les agents. Sa vision fonctionnaliste l'amène à considérer les 
effets du capital social. Il distingue les manifestations du capital à travers les droits et les obligations relevant d'un environnement social marqué par la confiance, la capacité de circulation de l'information au sein de la structure sociale, et l'existence de normes et de sanctions qui s'imposent et que respectent les membres d'une communauté. On notera que deux des formes de capital social précédemment définies (i.e. obligation et normes réciproques) ne posent pas ce dernier au fondement des relations sociales mais le présentent au contraire comme un dérivé de la structure sociale. La conception fonctionnaliste du capital social tend à obscurcir la notion, la présentant à la fois comme un "output" de la structure sociale et comme un élément structurant qui la détermine. Ce type de raisonnement accroît la confusion sur la notion (Fukuyama, 1999 ; Woolcok, 2001) et alimente plus largement un raisonnement déterministe et tautologique sur l'explication des mécanismes de développement. On s'entend ainsi dire que le développement des sociétés s'explique par l'efficience de leurs structures sociales (en somme un degré de capital social élevé) et que les sociétés développées sont celles qui disposent de structures sociales efficientes, et inversement.

(iii) D'autres travaux abordent le capital social sous l'angle des ressources (Lin, 1995, 2001). Suggérant d'intégrer le capital social dans une théorie générale des ressources, Lin le définit comme une richesse potentielle incorporée dans la structure sociale et qui peut être (mais ne l'est pas nécessairement) mobilisée en cas de besoin. Le concept de capital social recouvrirait ainsi à la fois des ressources existantes et latentes liées à un réseau relationnel stable plus ou moins activé. Cette conception rejoint celle adoptée par Bourdieu pour lequel "le capital social que possède un agent particulier dépend de l'étendue du réseau des liaisons qu'il peut effectivement mobiliser et du volume du capital (économique, culturel ou symbolique) possédé en propre par chacun de ceux auquel il est lié". Ainsi, le capital social n'est autre que "l'ensemble des ressources actuelles ou potentielles qui sont liées à la possession d'un réseau durable de relations plus ou moins institutionnalisées d'interconnaissance et d'inter-reconnaissance" (Bourdieu, 1980, p.2.).

Cet exercice de définition conceptuelle du capital social montre la difficulté à cerner précisément la notion. Une appréhension concrète du concept invite à décliner les différentes formes du capital social.

- Le capital social, un concept multiforme

Le capital social désigne différents aspects des rapports sociaux. On distingue dans la littérature trois typologies déclinant 
ces divers aspects. A travers cet effort de caractérisation du capital social, la structure sociale est appréhendée aussi bien du point de vue microéconomique que macroéconomique.

(i) Collier (1988) distingue entre capital social gouvernemental et civil. Le premier fait référence aux institutions ou organisations mises en place par l'Etat ou le secteur public alors que le second fait référence aux institutions ou organisations émanant de la société. On identifie alors dans chacune de ces formes, deux formes de capital social : les institutions en tant que règles et les organisations au sein desquelles est assuré le respect de ces règles.

Ces deux formes de capital social sont interdépendantes. Les travaux de Narayan (1999), Dasgupta (2000) et Grootaert (1998), Woolcok et Narayan (2000) montrent en effet que capital social civil et gouvernemental sont dans certains cas complémentaires ou substituables. Par exemple, la substitution du capital social civil au capital social gouvernemental opère lorsque le fonctionnement de l'Etat est défaillant. On observe ce phénomène en situation de conflit violent (i.e. guerre civile) où les groupes dominants font valoir leur pouvoir. A l'inverse, une complémentarité entre capital social gouvernemental et civil s'instaure lorsque l'Etat accomplit sans perturbation sa fonction de "gouvernance". Dans ce cas, les liens entre les structures gouvernementales et civiles renforcent la cohésion sociale. Dans le même ordre d'idée, Putnam (1993) expose que l'engagement civique renforce la stabilité des institutions étatiques de la même façon que les institutions gouvernementales créent un environnement favorable à l'engagement civique.

Ces développements conceptuels tendent à présenter le capital social comme la matière première d'une société, nécessaire au bon fonctionnement des institutions. Cette approche, qui souligne le degré d'interaction entre l'environnement socio-politique et le secteur privé, attire également l'attention sur une autre nature du capital social.

(ii) Uphoff (2000) différencie entre capital social structurel (entendu comme l'ensemble des institutions visibles) et capital social cognitif (ou subjectif c'est-à-dire qui renvoie aux processus mentaux des individus). En clair, le premier désigne les structures dans lesquelles agissent les agents (i.e. les organisations) alors que le second fait référence aux valeurs, normes, croyances etc. qui prédisposent les agents à la coopération. Pour désigner cette même distinction de nature sur le capital social, Krishna (2000) parle respectivement de capital social institutionnel et relationnel.

Une conception globale du capital social invite à un recoupement entre les quatre formes précédemment identifiées. Le tableau suivant présente quelques exemples de capital social.

36 Economie et Institutions $-n^{\circ} 6$ et $7-1^{\text {er }}$ et $2^{\text {e }}$ semestres 2005 
Tableau 1 Les formes de capital social

\begin{tabular}{|l|l|l|}
\hline & $\begin{array}{l}\text { Capital social } \\
\text { structurel }\end{array}$ & $\begin{array}{l}\text { Capital social } \\
\text { cognitif }\end{array}$ \\
\hline $\begin{array}{c}\text { Capital social } \\
\text { civil }\end{array}$ & $\begin{array}{l}\text { Club, association, } \\
\text { ONG, communauté .... }\end{array}$ & $\begin{array}{l}\text { Normes, croyances, } \\
\text { valeurs ... }\end{array}$ \\
\hline $\begin{array}{l}\text { Capital social } \\
\text { gouvernemental }\end{array}$ & $\begin{array}{l}\text { Etat, police, } \\
\text { tribunaux ... }\end{array}$ & Lois, régime politique ... \\
\hline
\end{tabular}

Source : d'après Sirven, 2000.

Cette vision intégrée accorde de l'importance aux interdépendances entre les différentes formes de capital social. Si elle invite à considérer la capacité des structures sociales à élaborer des règles facilitant l'établissement de liens entre agents, elle n'indique rien sur la densité des liens établis.

(iii) Concernant la nature des liens, il faut citer l'approche popularisée par la Banque Mondiale en 2000 sur le capital social qui unit, qui lie et qui relit ("bonding", "linking", bridging").

Les liens de type "bonding" unissent des individus au sein d'une même communauté. Les liens sont encore qualifiés d'horizontaux. Dans ce type de structures, les agents sont de statut identique : ils appartiennent au même groupe. On y range explicitement les relations familiales et amicales ainsi que les réseaux sociaux (communauté d'individus, organisation etc.). Les liens de type "linking" caractérisent des interactions entre des agents appartenant à des groupes différents. Ces liens sont de nature verticale. Dans ce type de relation, les agents occupent des places (ou de statuts) différents. L'idée de réseau étendu illustre bien ce type de liens (réseau de réseau). Enfin, dans les relations de type "bridging", les agents sont distants les uns des autres. Cette dernière notion désigne à la fois leur éloignement physique (les liens de type bridging sont extra-territoriaux) et la discontinuité dans l'activation du lien (l'idée de bridging rend compte du caractère latent du capital social, apparenté dans ce cas à une ressource non activée). Le "bridging social capital" désigne un réseau virtuel.

En résumé, cette synthèse sur la nature des liens doit être rapprochée de la vision dichotomique de Granovetter (1973) distinguant les liens forts des liens faibles. Le capital social de type bonding et linking peuvent être saisis comme des liens forts, alors que le capital social de type bridging appartient à la catégorie des liens faibles.

C'est cette dernière approche sur la nature des liens que nous retiendrons dans le cadre de nos réflexions sur les coordinations locales et le développement. Nous montrerons notamment en quoi cette approche du capital social s'articule avec l'économie de proximité. 

approches théoriques du capital social pour une théorisation du développement local

L'économie de proximité et l'approche du capital social présentent des démarches d'analyse similaires. Ces deux cadres d'analyse visent à caractériser les modalités de coordination entre les agents. Ils proposent chacun une analyse de la nature des liens tissés entre les acteurs. Si ces deux courants ont en commun d'aborder la question de l'impact de ces coordinations locales sur le développement, l'approche du capital social se focalise davantage sur la densité des liens. Nous verrons que la procédure d'activation des liens repose sur des logiques que permet d'appréhender la proximité (1). S'agissant de la force des liens, nous montrerons que les deux courants divergent et que les conclusions théoriques du capital social enrichissent celles de l'économie de proximité (2).

\subsubsection{Le capital social, un capital activé par de liens sociaux}

Les développements théoriques précédents mettent en évidence le caractère social du capital social. Il s'agit maintenant de se demander si le capital social recèle les propriétés du capital. Le capital social, s'il s'apparente à du capital, doit pouvoir être appréhendé en termes de stock et de flux (i) et doit également être accumulable (ii).

(i) Le capital social génère des revenus

Dès les années 1960, les économistes proposent un élargissement du concept de capital (Schultz, 1961 ; Becker, 1964). Ils entendent par là saisir sous cette dénomination d'autres facettes du capital que ses seuls aspects matériels. La littérature évoque à ce titre les termes de capital humain et de capital social. Toutefois, si le capital humain renvoie à des caractéristiques individuelles (i.e. niveau de qualification des individus), le capital social relève du réseau de relation des individus. En d'autres termes, on veut signaler que les individus (ou groupes d'individus) peuvent profiter de ressources qui se constituent à travers leurs relations aux autres (individus ou groupes).

L'analyse économique définit le capital comme un ensemble de biens qui pourvoient des revenus à ceux qui les détiennent. Les biens possédés (i.e. les ressources sociales) constituent le stock de capital alors que les revenus (i.e. les effets de ces ressources sur la situation économique de l'agent) correspondent à des flux. La notion de stock renvoie à la quantité de biens que possède un agent donné. Toute la question est de savoir si cette quantité de biens est 
invariante ou non et si leurs effets sont positifs c'est-à-dire génèrent un profit.

Les travaux sur le capital social montrent qu'il est susceptible d'évoluer dans le temps (le stock de capital n'est donc pas invariant) et que c'est un moyen, pour les agents de corriger sa distribution de revenus. Pour qu'une relation sociale soit profitable à un agent, il faut qu'elle lui donne accès à un certain niveau de capital social. Autrement dit, l'investissement d'un individu dans ses relations aux autres doit lui permettre d'améliorer sa situation économique. On peut citer l'exemple d'un individu qui s'appuierait sur son réseau relationnel pour arriver à ses fins (i.e. favoriser son insertion professionnelle). Comme on le voit ici, le capital social est fonction de la capacité de l'agent à tirer partie de son réseau. Mais pour que ces relations soient utiles ou potentiellement utiles, il est nécessaire qu'elles soient elles-mêmes porteuses de ressources. Un individu peut, en effet, disposer d'un certain niveau relationnel (c'est-à-dire de capital social) sans que ce dernier lui permette d'augmenter son propre niveau de ressources. Si l'on reprend le cas de l'individu en situation de recherche d'emploi, le réseau relationnel dans lequel il s'inscrit peut ne lui être d'aucune utilité en matière d'insertion professionnelle.

En clair, un capital social élevé peut avoir peut avoir des effets marginaux selon la disponibilité des ressources existant dans les relations réticulaires d'un individu. Ces propos se rattachent à une conception quelque peu déterministe de la structuration sociale appréhendée à travers la qualité de capital social auquel les individus ont accès à travers leurs réseaux de relations. Dans sa théorie des ressources sociales, Lin (1995) montre que les positions sociales d'origine conditionnent l'accès au capital social et à son usage. A l'issue de ce qui précède, on convient à l'idée que le niveau de capital social (tout comme ses effets d'ailleurs) résulte de l'activation de liens sociaux. L'accumulation de capital social s'effectue à travers la mobilisation des relations sociales.

(ii) Le capital social est accumulable

En tant que capital, le capital social doit être accumulable. Les développements précédents nous amènent à concevoir que la productivité du réseau (en termes de ressources sociales) ne dépend pas que du lien établi entre des agents ou groupes d'agents mais de l'activation de ce lien. Autrement dit, la création et l'accumulation de capital social impliquent que les liens entre individus soient mobilisables et mobilisés.

L'analogie entre le capital social et les autres formes de capital (le capital humain notamment) réside dans le fait qu'il peut être à la fois acte de consommation et d'investissement. En effet, 
lorsqu'un agent active un lien social, en recourant par exemple au service d'une connaissance, il consomme du capital social. Mais en même temps, comme il entretient la relation, il procède également à un investissement social. Cette interaction sociale augmente la force du lien entre les deux individus insérés dans la relation en générant de nouvelles règles de rapport. Le demandeur de service est placé dans une relation d'obligation et de dette par rapport l'offreur. Ce dernier, lorsqu'il consent à rendre service témoigne une preuve de confiance au bénéficiaire. Ce faisant, cette interaction sociale contribue à l'accumulation de capital social (ici cognitif).

Ces éléments d'analyse nous invitent à souligner la particularité du capital social par rapport au capital physique. A l'inverse de ce dernier, le capital social ne se déprécie pas avec l'usage. Bien au contraire, il se délite lorsque les liens sociaux ne sont pas entretenus ${ }^{8}$. En conséquence, l'accumulation de capital social dépend étroitement non seulement de la qualité des relations entre les agents mais également de leur régularité. Les liens sociaux doivent être continuellement réactivés, sinon ils perdent en efficacité. Si l'on rapporte cette dernière conclusion aux différentes formes de capital social, on admet que les liens sociaux de type bonding ou linking nécessitent d'être constamment renforcés pour que le capital social soit effectivement une ressource utile. En revanche, le capital social de type bridging n'implique pas une activation régulière, ni intense des liens. Il faut à ce titre observer la spécificité de cette forme de capital social, puisque par essence, elle repose sur l'établissement de liens faibles. La nature des liens élaborés entre les agents peut alors être caractérisée par leur densité et appréhendée en termes de proximité. Le tableau ci-dessous synthétise notre propos.

Tableau $2 \quad$ Nature et densité des liens

\begin{tabular}{|c|c|c|c|}
\hline & & Densité du lien & Degré de proximité \\
\hline \multirow{3}{*}{ 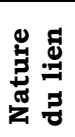 } & Bonding & Activation forte du lien & \multirow[b]{2}{*}{ Proximité forte } \\
\hline & Linking & Activation forte du lien & \\
\hline & Bridging & Activation faible du lien & Proximité faible \\
\hline
\end{tabular}

En résumé, le capital social, pour être efficacement mobilisé, doit s'appuyer sur une dynamique de liens sociaux particulière. Il repose sur une certaine densité de rapports, ce qui suppose que les agents s'inscrivent, d'une certaine façon, dans des relations de

8 La capacité d'entretien du lien varie selon un nombre important de critères (fonction eux-mêmes des agents et de leurs habitudes relationnelles) qui rendent impossible de définir la vitesse avec laquelle se déprécie le capital social.

40 Economie et Institutions $-n^{\circ} 6$ et $7-1^{\text {er }}$ et $2^{\text {e }}$ semestres 2005 
proximité. Ces structures relationnelles sont plus ou moins denses et cohésives. Les différentes formes de liens qui les constituent se distinguent alors par leur vigueur et leur puissance. C'est en ce sens que nous parlons de force des liens.

\subsubsection{La force des liens}

Dans cette section, nous chercherons à décrire la force des liens de proximité. Il faut entendre par là le spectre des configurations marqué par un gradient de proximité (faible à forte). Notre raisonnement se déroulera en deux temps. (i) Nous commencerons par décrire quelles règles cimentent ces différentes relations de proximité, (ii) puis nous chercherons à en apprécier la portée. Ces éléments de réflexion nous donneront matière à alimenter notre théorisation du développement local.

- La vigueur des liens : quelles règles président aux relations de proximité ?

Nous avons souligné précédemment que le capital social doit être entretenu à travers l'activation de liens sociaux. Une bonne compréhension de la procédure d'activation des liens nous invite à l'analyse des règles qui président aux relations entre agents. La proximité permet de spécifier ces règles.

Les liens de type bonding caractérisent des relations affectives, des affinités sociales. Ces liens sont de type communautaire et sont porteurs d'une logique d'exclusion des membres extérieurs à la communauté. Ils tendent vers une "fermeture relationnelle" (Coleman, 1988). L'appartenance à une même communauté rend compte de ce que les agents adhèrent au même système de représentation. Et dans ce type de liens, la confiance interpersonnelle est élevée. Les agents élaborent leur propres règles et organisent leur contrôle. Ces liens intra-groupe expriment un degré de proximité fort entre les agents. Ici, c'est le registre institutionnel de la proximité qui prévaut (logique de similitude).

Le capital social de type linking nécessite une activation régulière des liens sociaux. Ces liens inter-groupe doivent par conséquent être continuellement réaffirmés. Le processus d'échange entre les agents implique la fréquence des interactions. Ils se caractérisent par des transactions de réciprocité qui oblige à la poursuite des échanges. C'est bien l'effectivité des relations qui est ici soulignée. Elle fait référence au registre organisationnel de la proximité (logique d'appartenance). Comme nous l'avons déjà mentionné, la fréquence des interactions tend à déboucher sur la convergence des représentations (élaboration de normes communes, réciprocité) et peut ainsi nourrir la dimension institutionnelle de la 
proximité. Dans le capital social de type linking, c'est donc d'abord le registre organisationnel de la proximité qui est au fondement des relations mais il n'est pas exclu que la logique institutionnelle de la proximité contribuent à les renforcer. Nous noterons que la distinction entre les liens de type bonding et linking n'est pas toujours aisée et que les deux formes de relations sociales peuvent parfois se recouvrir.

Cependant, si les liens de type bonding et linking reposent sur des structures relationnelles cohésives qui créent et augmentent le sentiment d'appartenance et de solidarité entre les membres du groupes, dans les liens de type bridging, prévalent d'autres logiques. La fréquence des contacts n'est pas une condition nécessaire à la mobilisation du capital social. La procédure de mise en accord entre les agents est ponctuelle et discontinue. Les règles respectées s'apparentent alors à une convention sans engagement réciproque de leur part. Dans ce cas, ce ne sont pas des logiques de proximité qui œuvrent dans ce type de relation. Le tableau suivant résume l'ensemble de ces propos.

Tableau 3 Nature des liens sociaux et logiques de proximité

\begin{tabular}{|l|l|l|l|}
\hline Nature du lien & Motivation du lien & $\begin{array}{l}\text { Nature et propriétés } \\
\text { des règles ratifiées }\end{array}$ & Logique de proximité \\
\hline Bonding & $\begin{array}{l}\text { Morale } \\
\text { Investissement social } \\
\text { non intentionnel }\end{array}$ & Introjection de valeur & Logique de similitude \\
\hline Linking & $\begin{array}{l}\text { Intérêt } \\
\text { Investissement social } \\
\text { intentionnel }\end{array}$ & $\begin{array}{l}\text { Réciprocité et obligation } \\
\text { Intérêt } \\
\text { intentissement social }\end{array}$ & $\begin{array}{l}\text { Logique d'appartenance } \\
\text { (mais n'exclut pas } \\
\text { la logique de similitude) }\end{array}$ \\
\hline engagement réciproque & $\begin{array}{l}\text { Logique de relation de } \\
\text { distance } \\
\text { (liens de proximité } \\
\text { inexistants) }\end{array}$ \\
\hline
\end{tabular}

Nous avons considéré jusqu'à présent le capital social comme une grille d'analyse des liens sociaux. Il convient maintenant de s'intéresser à la portée concrète des liens sociaux auxquels on prête une incidence non négligeable sur la performance économique locale.

- La puissance des liens : quelle incidence des liens de proximité sur la performance économique locale?

Nous nous proposons de réfléchir ici à l'impact des liens sociaux sur le processus de développement. On verra que la force des liens n'est pas la même selon le type de capital social qu'ils activent. Cependant, avant de prétendre que le capital social affecte le développement, il faut se demander si on peut véritablement en isoler les effets propres. Cette question est discutée dans la littérature.

42 Economie et Institutions $-n^{\circ} 6$ et $7-1^{\text {er }}$ et $2^{\text {e }}$ semestres 2005 
Le capital social n'est pas la seule ressource dont dispose les agents. Ce n'est qu'une ressource parmi d'autres. L'enjeu est donc d'isoler la valeur relative et les effets spécifiques du capital social. L'exercice n'est pas aisé, car le capital social est fongible. Cela signifie qu'il peut se transformer en d'autres formes de capital. A titre d'exemple, lorsqu'une aide financière est proposée à un individu par un membre de son réseau relationnel, le capital social se transforme en capital économique. De la même façon, lorsqu'un individu se sert de son réseau pour accéder à une formation, il tend à améliorer son niveau de qualification initiale. Le capital social se trouve ainsi transformé en capital humain. On voit alors que le caractère fongible du capital social le rend interdépendant des autres formes de capital. Il peut être, pour reprendre les termes de (Adler et Kon, 1999), un substitut ou un complément à d'autres ressources. Si pour certains auteurs, le capital social n'a pas de contenu propre ${ }^{9}$, d'autres comme Putnam $(1993,1995)$ s'attachent à le mesurer mais également à en évaluer les effets.

Pour identifier les effets du capital social, il faut parvenir à mesurer de quelle manière le capital social affecte la situation économique (ou bien-être) des agents. Plusieurs études empiriques portent sur la mesure du capital social. Certaines se centrent sur le niveau individuel. Elles visent à identifier le niveau de ressources auquel accède un individu en mobilisant son réseau (Lin, 1982 ; Burt, 1992 ; Forsé, 1997). D'autres analyses se concentrent sur le niveau collectif. Le capital social est celui du groupe. C'est alors un bien collectif que partage le groupe. Ces deux formes de capital social (et leurs effets) doivent être considérées de manière différente. Si l'on se situe dans la conception individuelle du capital social, on l'associe à un actif qui influe sur le revenu de l'agent. Par contre, si l'on adopte une vision plus globale, on appréhende le capital social comme un indicateur d'efficacité collective. Dans ces conditions, on s'attache au rôle du capital social dans les activités de production et aux externalités qu'il produit, ce qui permet de s'interroger sur les trajectoires de croissance et de développement des espaces dotés en capital social. C'est dans ce dernier registre d'analyse que nous nous inscrivons, s'agissant de nos réflexions sur le développement.

L'importance du capital social comme variable explicative de la performance économique locale est montrée à travers les externalités qu'il produit. Nous avons montré antérieurement (Cf. partie 1) que les externalités générées par le capital social sont relatives à la circulation de l'information et à l'action collective. Il

9 Pour Bourdieu (1980, 1987), par exemple, le capital social est un multiplicateur de capital. Il est en cela second par rapport aux autres formes de capitaux. C'est un "méta- capital". 
s'agit maintenant de comprendre quelles formes de liens sociaux favorisent ces externalités.

De ce point de vue, on conçoit que les liens de proximité forts (qu'ils soient de type bonding ou linking) favorisent l'accumulation d'informations sur les individus. En revanche, dans un capital social de type bridging, les liens entre individus étant par nature des liens de proximité faible, ils ne peuvent constituer un canal de diffusion approprié pour des informations relatives au comportements individuel, ni contribuer à l'émergence d'actions collectives. Les liens faibles se révèlent par contre être une bon vecteur d'informations relatives à la connaissance de l'environnement. Les agents, précisément non inscrits dans des logiques de proximité, détiennent des informations diversifiées qu'ils s'échangent. Or dans les processus de développement, ce qui est mis en exergue, c'est la capacité des agents à maîtriser les paramètres de l'environnement dans lequel ils opèrent. On voit ici, pour reprendre la terminologie de Granovetter (1973), la "force des liens faibles".

Notre analyse des liens sociaux nous permet de considérer de quelle manière les coordinations locales influencent le développement. On admet dès lors, que le capital social puisse tenir une place privilégiée dans les analyses du développement. L'articulation entre l'économie de proximité et l'approche du capital social nous paraît essentielle à la compréhension des déterminants de la performance économique locale. Elle nous paraît, à ce titre, fondamentale pour une théorisation du développement local.

\section{Conclusion}

L'apport de notre contribution tient dans l'articulation de l'économie de proximité et de l'approche du capital social afin de mieux cerner les mécanismes à l'œuvre dans les processus de développement local. Les contributions de ces deux cadres théoriques pour l'analyse des coordinations locales nous ont permis d'appréhender l'importance des dynamiques sociales dans les processus de développement. Notre analyse montre bien l'intérêt qu'il $\mathrm{y}$ a à combiner ces deux programmes de recherche pour aboutir à une théorisation complète du développement local.

En effet, l'économie de proximité met surtout l'accent sur la force de liens forts dans les dynamiques de développement territorial. Or, un excès de localisme peut être néfaste au développement. Les coordinations locales peuvent, en effet, enfermer les territoires dans des trajectoires de blocage ("lock in"). L'un des apports de l'approche du capital social est de montrer la force des liens faibles. On montre alors que ce ne sont pas tant les relations de proximité qui importent dans les logiques de développement que les relations de distance. Ces liens extra-territoriaux et non nécessairement activés rompent

44 Economie et Institutions $-\mathrm{n}^{\circ} 6$ et $7-1^{\text {er }}$ et $2^{\text {e }}$ semestres 2005 
avec les caractéristiques organisationnelles et institutionnelles que l'économie de proximité prête aux coordinations efficaces. Si l'économie de proximité ne nie pas l'impact négatif que peuvent parfois avoir les liens forts sur les dynamiques de développement, elle ne va pas jusqu'à montrer l'importance des liens faibles dans les dynamiques de développement.

Notre démarche théorique d'explicitation des mécanismes de développement auxquels renvoient les formes sociales organisées, nous a menés à souligner essentiellement le caractère positif des externalités générées par les coordinations locales. Si ce point fait débat dans les deux champs théoriques mobilisés, il importe de confronter à des réalités de terrain l'hypothèse du rôle des formes sociales organisées dans le développement local pour mieux saisir leur portée réelle.

\section{Références}

Adler P. et S.W. Kon, (1999), "Social capital : the good, the bad, and the ugly", Research Paper, Dept. Of management and organization, University of Southern California, Los Angeles.

Angeon V. et A. Caron, (2004), "Valorisation de ressources et attractivité des territoires. L'environnement, facteur de spécification des ressources", Montagnes Méditerranéennes, 20 (décembre), pp. $55-68$.

Arrow K., (1974), The Limits of Organization, New York, W. W. Norton \& Company.

Ballet J. et R. Guillon, (2003), Regards croisés sur le capital social, Paris, Paris, Ed. L'Harmattan, Coll. Ethique économique.

Becker G.S. et K.M. Murphy, (2000), Social economics: market behavior in a social environment, Harvard University Press.

Becker G.S., (1964), Human capital, New York, Columbia University Press.

Bellet M., G. Colletis, B. Lecoq, Y. Lung, B. Pecqueur, A. Rallet et A. Torre, (1992), "Et pourtant ça marche ! (quelques réflexions sur l'analyse du concept de proximité)", Revue d'Economie Industrielle, 61, 3è trimestre, pp. 111-128.

Bellet M., G. Colletis et Y. Lung, (1993), "Economie des proximités", Revue d'Economie Régionale et Urbaine, numéro spécial $\mathrm{n}^{\circ} 3$, ADICUEER pp 357-606.

Bellet M., T. Kirat et C. Largeron, (1998), Approches multiformes de la proximité, Eds. Hermès. Paris. 343pp.

Bourdieu P., (1980), "Le capital social : notes provisoires", Actes de la recherche en sciences sociales, 31, pp. 2-3.

Bourdieu P., (1987), "What makes a social class? On the theoretical and practical existence of groups", Berkeley Journal of Sociology 32, pp. 1-17. 
Burt R.S., (1992), Toward a Structural Theory of Action, New York, Academic Press.

Burt R.S., (2000), "The network structure of social capital", Research in organizational behaviour, 22, pp. 355-373.

Calame P., (1991), "Projets de ville, projets de vie, esquisse d'une théorie de l'action collective", Actes, 5 (Octobre 1991).

Caron A. et A. Torre, (2002), "Les conflits d'usage dans les espaces ruraux : une analyse économique", in Perrier-Cornet Philippe (dir.), A qui appartient l'espace rural ?, Editions de l'Aube Datar, pp.49-78

Coleman J.S., (1988), "Social capital in the creation of human capital", American journal of sociology, vol. 94, pp. S95-S120.

Coleman J., (1990), Foundations of social theory, Cambridge, Harvard University Press.

Colletis-Wahl K. et B. Pecqueur, (2001), "Territories, development and specific resources: what analytical framework ?", Regional Studies, 35 (5), pp; $449-459$.

Collier P., (1998), "Social capital and poverty", Social capital Initiative Working paper, World Bank, Social Development Department, Washington, D.C. Processed.

Cote S. et T. Healy, (2001), The well being of nations. The role of human and social capital, Paris, Organisation for Economic Cooperation and Development.

Dasgupta P., (2000), "Economic Progress and the Idea of Social Capital", in P. Dasgupta et I. Serageldin (eds.), Social Capital: A Multifacted Perspective, World Bank, Washington DC.

Dasgupta P., (1999), "Economic progress and the idea of social capital" in P. Dasgupta et I. Serageldin, (eds.), Social Capital: A Multifacted Perspective, World Bank, Washington DC., pp. 325-424.

Durlauf S.N., (2002), "On the Empirics of Social Capital", Economic Journal, 112 (483), pp. F459-479.

Durlauf S. et Fafchamps M., (2004), "Social capital", Working paper, University of Wisconsin-Madison.

Dupuy C. et A. Torre (2000), "Confiance et coopération au sein des réseaux spatialisés d'entreprises", dans J.-P. Gilly et A. Torre (dir.), Dynamiques de proximité, Paris, Ed. L'Harmattan, coll. Emploi, Industrie et Territoire, pp.59-95.

Dupuy C. et A. Torre, (1998), "Liens de proximité et relations de confiance : le cas de regroupements localisés de producteurs dans le domaine alimentaire" dans M. Bellet, T. Kirat, et C. Largeron (Coord.) Approches multiformes de la proximité, Paris, Ed. Hermès, pp. 175192.

Forsé M., (1997), "Les réseaux sociaux", L'Année Sociologique, 47(1). Fukuyama F., (1999), "Social capital and civil society", IMF Conference on second generation reforms, $12 \mathrm{p}$.

Gilly J.-P. et A Torre, (2000), Dynamiques de proximité, Paris, Ed. L'Harmattan. Coll. Emploi, industrie et territoire, 301p.

46 Economie et Institutions $-\mathrm{n}^{\circ} 6$ et $7-1^{\mathrm{er}}$ et $2^{\mathrm{e}}$ semestres 2005 
Greffe X., (2002), Le développement local, Bibliothèque des territoires, Ed. de l'aube DATAR, Paris.

Granovetter M., (1973), "The strength of weak ties", American Journal of Sociology, 78(6), pp. 1360-1380.

Grootaert C., (1998), "Social capital : the missing link ?" Social capital initiative, Working paper $\mathrm{n}^{\circ} 3$, The World Bank, 22p.

Grossetti M., (1998), "La proximité en sociologie : une réflexion à partir des systèmes locaux d'innovation", dans M. Bellet, T. Kirat et C. Largeron (Coord), Approches multiformes de la proximité, Paris, Eds. Hermès, pp 83-100.

Hadenius A. et F. Ugla, (1996), "Making civil society work, promoting democratic development. What can states and donors do ?", World Development, 24(10), pp. 1621-1639.

Helliwell J.F. et R.D. Putnam, (2000), "Economic Growth and Social Capital in Italy", in P. Dasgupta et I. Serageldin, (eds.), Social Capital: A Multifacted Perspective, World Bank, Washington DC., pp. 253268.

Knack S. et P. Keefer, (1997), "Does Social Capital Have an Economic Payoff? A Cross-Country Investigation", Quarterly Journal of Economics, 112 (4), pp. 1251-1288.

Krishna A., (2000), "Creating and harnessing social capital", in P. Dasgupta et I. Serageldin (eds.), Social Capital: A Multifacted Perspective, World Bank, Washington DC., pp. 71-93.

Krishna A., (2001), "Moving from the Stock of Social Capital to the Flow of Benefits: The Role of Agency", World Development, 29(6), pp. 925-943.

Lévesque M. et D. White (1999), "Le concept de capital social et ses usages", Lien social et politiques - RIAC, 41 (Printemps 1999), pp. 2333.

Lin N., (2001), "Building a theory of social capital" in N Lin, K.S. Cook, et R.S. Burt (eds), Social capital: theory and research Eds, Paperback.

Lin N., (1995), "Les ressources sociales : une théorie du capital social", Revue Française de sociologie, 36, pp. 685-704.

Lin N. Y. Fu et, M. Hsung, (2001), "The position generator: measurement techniques for investigations of social capital", in N. Lin N., K. Cook K. et R. Burt (eds), (2001), Social capital. Theory and research, New York, Adline De Gruyter.

Marsden P. et N. Lin, (1982), Social Structure and Network Analysis, Beverly Hills, CA: Sage.

Narayan D., (1999), "Bonds and bridges : social capital and poverty", Policy Research Working Paper, World Bank, Poverty reduction and economic management network, Washington, D.C. Processed.

Narayan D. et L. Pritchett, (1999), "Cents and sociability: household income and social capital in rural Tanzania", Economic development and social change, 47(4), pp. 871-897. 
North D.C., (1990) Institutions, Institutional Change and Economic Performance. Cambridge, Cambridge University Press.

North, D.C., (1986), "The New Institutional Economics." Journal of Institutional and Theoretical Economics, 142(1986): 230-237.

Paldam M. et G.T. Svendsen, (2000), "An essay on social capital: looking for the fire behind the smoke", European journal of political economy, 16, pp. 339-366.

Pecqueur B., (1996) Dynamiques territoriales et développement économique, Paris, Ed. L'Harmattan.

Pecqueur B., (1989), Le développement local, Paris, Syros, collection Alternatives économiques.

Pecqueur B. et J.B. Zimmerman, (2004), Economie de proximités, Paris, Ed. Hermès.

Peyrache-Gadeau V. et B. Pecqueur, (2002), "Les ressources patrimoniales : une modalité de valorisation par les milieux innovateurs de ressources spécifiques latentes ou existantes", Neuchâtel, 26-27 avril 2002.

Perroux F., (1964), L'économie du XXème siècle, Paris, PUF, 2ème édition.

Putnam R., (1993), Making democracy work : civic traditions in modern Italy, Princeton, Princeton University Press.

Putnam R., (1995), "Tuning in, tuning out: the strange disappearance of social capital in America", Political Science and Politics, December, pp. 664-683.

Putnam R.D., (2000), Bowling alone. The collapse and revival of American community, New York, Simon Schuster publishers.

Rallet A., (1999), "L'économie de proximités", Ecole-Chercheurs INRA Economie spatiale et régionale, Le Croisic, 8-10 décembre, 24p.

Requier-Desjardins D., (2003), "Le capital social dans la théorie économique : actif privé ou bien public? Le point sur quelques contributions récentes", dans J. Ballet et R. Guillon (éds.), Regards croisés sur le capital social, L'Harmattan, Coll. Ethique économique, Paris, pp. 39.

Requier-Desjardins D., (2000), "On some contributions on the definition and relevance of social capital", Cahier du C3ED, 18p.

Rupasingha A., S.J. Goetz et D. Freshwater, (2002), "Social and Institutional Factors as Determinants of Economic Growth: Evidence from the United States Counties", Papers in Regional Science, 81 (2), pp. 139-155.

Serageldin I. et C. Grootaert, (2000), "Defining social capital: an integrated view", in P. Dasgupta et I. Serageldin, eds., Social Capital: A Multifacted Perspective, World Bank, Washington DC., pp. 40-58.

Schultz, T.W., (1961), "Investment in human capital" American Economic Review, 51(1), pp.1-17.

Sirven N., (2003), "L'endogénéisation du rôle des institutions dans la croissance ou la (re)découverte du capital social" dans J. Ballet et R.

48 Economie et Institutions $-n^{\circ} 6$ et $7-1^{\text {er }}$ et $2^{\text {e }}$ semestres 2005 
Guillon, 2003, Regards croisés sur le capital social, Paris, Ed. L'Harmattan, Coll. Ethique économique, pp. 57-90.

Sirven N., (2000), "Capital social et développement: quelques éléments d'analyse ", Centre d'Economie du Développement, Document de travail $n^{\circ} 57$, Bordeaux, 26p.

Teisserenc P., (2002), Les politiques de développement local, Paris, Economica.

Torre A. et A. Caron, (2002),"Conflits d'usage et de voisinage dans les espaces ruraux", Sciences de la Société, 57, pp.95-113.

Uphoff N., (2000), "Understanding social capital: learning fron the vanalysis and experience of participation", in P. Dasgupta et I. Serageldin (eds.), Social Capital: A Multifacted Perspective, World Bank, Washington DC.pp. 215-249.

Woolcok M. et D. Narayan, (2000), "Social capital: implication for development theory, research and policy", The World Bank Research Observer, 15(2), pp. 225-49.

Woolcok M., (2001), "La place du capital social dans la compréhension des résultats sociaux et économiques", DRHC/OCDE.

Zimmermann J.B., (1998) "Nomadisme et ancrage territorial: propositions méthodologiques pour l'analyse des relations firmesterritoires", Revue d'Economie Régionale et Urbaine, 2.

Zimmermann J.B, J.P. Gilly, J. Perrat, B. Pecqueur et F. Rychen, (1998), Construction territoriale et dynamiques productives, Etude pour le commissariat général du plan, décembre, $261 \mathrm{p}$. 